\title{
Estimativa da área foliar da batateira, cultivar Atlantic, utilizando dimensões lineares ${ }^{1}$
}

\author{
Evaluation of the potato plant leaf area, cultivar Atlantic, using linear measures
}

\author{
Camilo Busato $^{2 *}$, Paulo Cezar Rezende Fontes ${ }^{3}$, Heder Braun e Cristiani Campos Martins Busato $^{5}$
}

\begin{abstract}
Resumo - Objetivou-se com este trabalho estabelecer modelos matemáticos mais apropriados para estimar a área foliar da cultivar de batata Atlantic, utilizando-se o comprimento (C) e a largura (L) da folha. As avaliações foram realizadas em 270 folhas coletadas aleatoriamente no campo de produção da Universidade Federal de Viçosa, em duas épocas, aos 21 e 50 dias após a emergência das plantas. Em laboratório, foram mensurados o comprimento e a largura das folhas, além da área foliar por um integrador de área foliar. Os dados obtidos foram submetidos à análise de regressão, sendo a AF considerada como uma variável dependente, e o C, a L, o produto do C pela L (CL) e o quadrado do produto do $\mathrm{C}$ pela $\mathrm{L}(\mathrm{CL})^{2}$ como sendo variáveis independentes. A área foliar foi estimada com maior precisão usando as medidas de comprimento e largura, sendo: $\mathrm{AF}=0,369335^{* *} \mathrm{CL}+10,6923\left(\mathrm{R}^{2}=0,92\right)$, aos $21 \mathrm{DAE}$ e AF $=0,470971 * * \mathrm{CL}-16,3432$ $\left(\mathrm{R}^{2}=0,86\right)$, aos 50 DAE. Com os modelos propostos, a área foliar da batateira, cultivar Atlantic, pode ser estimada com as medidas de comprimento e largura obtidas diretamente no campo, em tempo real, de forma rápida e sem a necessidade de destruir as folhas e plantas.
\end{abstract}

Palavras-chave - Batata. Análise foliar. Métodos matemáticos.

\begin{abstract}
The objective of this research was to establish the appropriated mathematical model to estimate leaf area of potato cultivar Atlantic using the linear dimensions of the leaf: the length (C) and width (L). The evaluation was performed in 270 leaves, which were randomly collected from a field of Universidade Federal de Viçosa, 21 and 50 days after the plant emergence (DAE). In laboratory, the length, width and area of each leaf were measured by a leaf area meter (model LI 3100). The data were submitted to a regression analysis with the AF value as a dependent variable and the leaf length, width, the product of both $(\mathrm{CL})$, and the square product $(\mathrm{CL})^{2}$ values as independent variables. Potato plant LA was more precisely estimated using measures of length and width: $A F=0,369335 * * C L+10,6923\left(R^{2}=0,92\right)$ to 21 DAE and $\mathrm{AF}=0,470971^{* *} \mathrm{CL}-16,3432\left(\mathrm{R}^{2}=0,86\right)$ to $50 \mathrm{DAE}$. With the obtained model, AF of potato cultivar Atlantic can be estimated by measures obtained straight from the field, with no the need to damage the leaf or the plant.
\end{abstract}

Key words - Potato. Leaf analysis. Mathematical methods.

\footnotetext{
* Autor para correspondência

${ }^{1}$ Recebido para publicação em 04/12/2009; aprovado em 07/11/2010

Parte de pesquisa financiada pela FAPEMIG e CNPq

${ }^{2}$ Instituto de Defesa Agropecuária e Florestal do Espírito Santo/IDAF, Colatina-ES, Brasil, camilobusato@yahoo.com.br

${ }^{3}$ Universidade Federal de Viçosa/UFV, Viçosa-MG, Brasil, pacerefo@ufv.br

${ }^{4}$ Universidade Federal de Viçosa/UFV, Viçosa-MG, Brasil, hederbraun@hotmail.com

${ }^{5}$ Instituto Federal do Espírito Santo/IFES, Campus Itapina, Colatina-ES, Brasil, cristianicmartins@yahoo.com.br
} 


\section{Introdução}

A área foliar é característica importante na avaliação da eficiência fotossintética das plantas (TAIZ; ZEIGER, 2004), na determinação de danos bióticos e abióticos (LARCHER, 2000), na análise de crescimento, relacionado com o acúmulo de matéria seca, metabolismo da planta, maturação, qualidade e produção final das culturas (TAIZ; ZEIGER, 2004), diagnóstico nutricional (FONTES, 2001), além de servir para estimar a necessidade hídrica da cultura. Sua avaliação durante todo o ciclo da cultura é de extrema importância para que se possa modelar o crescimento e o desenvolvimento da planta e, em conseqüência, a produtividade e a produção total (TERUEL, 1995).

Os métodos de determinação da área foliar podem ser classificados como destrutivos ou não destrutivos e diretos ou indiretos (MARSHALL, 1968). Os métodos destrutivos são aqueles que exigem a retirada de partes da planta, o que pode não ser possível em alguns casos devido a particularidades do estudo em questão. Já os não-destrutivos não necessitam da retirada de suas partes, preservando, assim, a sua integridade e permitindo a continuidade das avaliações na mesma planta. Os métodos diretos são baseados em medidas realizadas diretamente nas folhas, enquanto os indiretos são baseados na correlação conhecida entre uma variável biométrica mensurável e área foliar real (KVET; MARSHALL, 1971).

Apesar de existirem inúmeras possibilidades para se determinar a área foliar, normalmente é utilizada a técnica destrutiva para medir a área da folha (BIANCO et al., 2002), no entanto, a busca de métodos fáceis de serem executados, rápidos e não destrutivos que estimem a área foliar com precisão torna-se importante para avaliar o crescimento das plantas nas condições de campo. Além disso, é útil em estudos sobre a atividade vegetal, como transpiração e fotossíntese que requerem um método de medição de área foliar não destrutivo. Pela facilidade e por ser não destrutivo, os mais utilizados são o comprimento ao longo da nervura principal, a largura máxima e as relações entre essas medidas (NASCIMENTO et al., 2002).

O uso das medidas lineares das folhas, tanto largura quanto comprimento, pode permitir o acompanhamento do crescimento e da expansão foliar da mesma planta, desde o início até o fim do ciclo (SILVA et al., 2008), reduzindo a variabilidade experimental associada com procedimentos de amostragens destrutivas (SILVA et al., 1998). Vários trabalhos têm relatado o uso de modelos matemáticos para estimar a área foliar, com base nas dimensões da folha de diversas espécies, sem destruir a amostra (BIANCO et al., 2003; DUARTE et al., 2009; MONTEIRO et al., 2005;
NASCIMENTO et al., 2002; PARTELLI et al., 2006; PIRES et al., 1999; QUEIROGA et al., 2003; SILVA et al., 1998; SILVA et al., 2008). Isso demonstra que esta técnica é uma importante ferramenta para a avaliação da área foliar de diversas espécies. O uso de medidas lineares, comprimento e largura das folhas, em modelos matemáticos para estimar a área foliar, é um método não destrutivo, de boa precisão e baixo custo, eliminando a disponibilidade de medidores de área foliar caros ou a realização de demoradas reconstruções geométricas, pois a área foliar é uma característica fácil de quantificar em tempo real, no próprio campo, sem necessidade de coletar a folha (FONTES; ARAÚJO, 2007). Assim, o objetivo deste trabalho foi estabelecer modelos matemáticos mais apropriados para estimar a área foliar da cultivar de batata Atlantic, utilizando-se medidas de comprimento e largura da folha.

\section{Material e métodos}

O experimento foi conduzido na Horta Nova de Pesquisa do Departamento de Fitotecnia da Universidade Federal de Viçosa (UFV), Viçosa-MG, com classificação climática, segundo Koppen (1948) tipo "Cwa", caracterizado pelo clima tropical de altitude, com chuvas no verão e secas no inverno, no período de outono/inverno (abril/julho de 2006). O solo utilizado nos experimentos é classificado como Podzólico Vermelho-Amarelo Câmbico (EMBRAPA, 2006) e suas características químicas e físicas foram determinadas pela análise de amostras das camadas de 0-20 cm de profundidade, realizada antes da instalação dos experimentos (TAB. 1).

O solo foi submetido à aração profunda com arado de aiveca e duas passagens de grade niveladora, com posterior sulcamento e aplicação de $200 \mathrm{~kg} \mathrm{ha}^{-1}$ de sulfato de amônio, $1800 \mathrm{~kg}$ de superfosfato simples, $384 \mathrm{~kg}$ de cloreto de potássio, $200 \mathrm{~kg}$ de sulfato de magnésio, $10 \mathrm{~kg}$ de bórax, $10 \mathrm{~kg}$ de sulfato de zinco, $10 \mathrm{~kg}$ de sulfato de cobre e $0,5 \mathrm{~kg}$ de molibdato de sódio.

O plantio dos tubérculos foi realizado no dia 03 de abril de 2006, utilizando-se batatas-semente filhas de caixa, com massa média de $70 \mathrm{~g}$, previamente brotadas em espaçamento de $0,75 \mathrm{~m}$ entre fileiras e 0,25 $\mathrm{m}$ entre plantas. A cultura foi conduzida seguindo as orientações de Fontes (2005) e foi realizado o controle de plantas daninhas e pulverizações contra pragas e doenças de acordo com a necessidade da cultura, além de irrigação por aspersão convencional.

Foram coletadas de forma aleatória, aos 21 dias após a emergência das plantas (DAE) (imediatamente antes da amontoa) e aos $50 \mathrm{DAE}$ (próximo à senescência das plantas), 
Tabela 1 - Características químicas e físicas do solo da camada de 0-20 cm de profundidade, antes da instalação do experimento com a cultivar Atlantic. Viçosa, UFV, 2006

\begin{tabular}{|c|c|}
\hline \multicolumn{2}{|c|}{ Características químicas } \\
\hline $\mathrm{pH}$ em água $-1: 2,5$ & 6,40 \\
\hline $\mathrm{N}-\mathrm{NO}_{3}^{-}\left(\mathrm{mg} \mathrm{kg}^{-1}\right)^{1}$ & 3,90 \\
\hline Matéria orgânica (MO) $\left(\mathrm{dag} \mathrm{kg}^{-1}\right)^{2}$ & 2,47 \\
\hline $\left.\mathrm{P}(\mathrm{mg} \mathrm{dm})^{-3}\right)^{3}$ & 73,70 \\
\hline $\left.\mathrm{K}(\mathrm{mg} \mathrm{dm})^{-3}\right)^{3}$ & 210,00 \\
\hline $\mathrm{Ca}^{2+}\left(\mathrm{cmol}_{\mathrm{c}} \mathrm{dm}^{-3}\right)^{4}$ & 4,80 \\
\hline $\mathrm{Mg}^{2+}\left(\mathrm{cmol}_{\mathrm{c}} \mathrm{dm}^{-3}\right)^{4}$ & 0,70 \\
\hline Acidez trocável $\left(\mathrm{Al}^{3+}\right)\left(\mathrm{cmol}_{\mathrm{c}} \mathrm{dm}^{-3}\right)^{4}$ & 0,00 \\
\hline Acidez potencial $(\mathrm{H}+\mathrm{Al})\left(\mathrm{cmol}_{\mathrm{c}} \mathrm{dm}^{-3}\right)^{5}$ & 3,14 \\
\hline Soma de bases (SB) $\left(\mathrm{cmol}_{\mathrm{c}} \mathrm{dm}^{-3}\right)$ & 6,04 \\
\hline CTC efetiva $(\mathrm{t})\left(\mathrm{cmol}_{\mathrm{c}} \mathrm{dm}^{-3}\right)$ & 6,04 \\
\hline CTC a pH 7,0 (T) $\left(\mathrm{cmol}_{\mathrm{c}} \mathrm{dm}^{-3}\right)$ & 9,18 \\
\hline Saturação de bases (V) (\%) & 66,00 \\
\hline \multicolumn{2}{|c|}{ Características físicas ${ }^{6}$} \\
\hline Areia grossa $\left({\left.\text { dag } \mathrm{kg}^{-1}\right)}^{-1}\right.$ & 5,00 \\
\hline Areia fina $\left(\mathrm{dag}_{\mathrm{kg}^{-1}}\right)$ & 23,00 \\
\hline Silte $($ dag kg-1) & 25,00 \\
\hline Argila (dag kg-1) & 47,00 \\
\hline Classificação textural & Argiloso \\
\hline
\end{tabular}

${ }^{1}$ Extrator $\mathrm{KCl} 1 \mathrm{~mol} \mathrm{~L}-1 ;{ }^{2} \mathrm{MO}=\mathrm{C} . \mathrm{Org}$ x 1,724 - Walkley-Black; ${ }^{3}$ Extrator Mehlich $1 ;{ }^{4}$ Extrator KCl 1 mol.L-1; ${ }^{5}$ Extrator Acetato de cálcio 0,5 mol.L $\mathrm{L}^{-1} \mathrm{pH} 7,0 ;{ }^{6}$ Método da "pipeta" segundo Embrapa

270 folhas de diferentes tamanhos e procedentes de plantas de batata (Solanum tuberosum L.) competitivas e livres de danos de ataques de pragas. Imediatamente após a colheita, as folhas foram individualizadas em saco plástico vedado, contendo gotas de água, e acondicionadas em caixas de isopor. Em laboratório, efetuou-se a medição do comprimento (C) e largura (L), avaliados individualmente, utilizando uma régua graduada em milímetros. O comprimento correspondeu à distância entre a base distal do pecíolo e a extremidade do folíolo terminal, assim como a largura à maior distância perpendicular ao eixo do comprimento, ambos expressos em centímetro $(\mathrm{cm})$. Também foi determinada a área de cada folha (AF), utilizando um integrador de área foliar modelo LI 3100 da LICOR.

Os dados obtidos foram submetidos à análise de regressão, utilizando-se o aplicativo SAEG (RIBEIRO JÚNIOR,2001). Ovalor daárea da folha(AF)foi considerado como variável dependente e o comprimento $(\mathrm{C})$, a largura (L), o produto comprimento x largura (CL) e o quadrado do produto do $\mathrm{C}$ pela $\mathrm{L}(\mathrm{CL})^{2}$, como variáveis independentes. Para o ajuste dos dados, os modelos estatísticos linear, exponencial e logarítmico foram selecionados para serem testados. Os modelos foram avaliados com base na lógica biológica, na sua simplicidade, no maior coeficiente de determinação $\left(\mathrm{R}^{2}\right)$ e na significância dos coeficientes de regressão, utilizando-se o teste $\mathrm{t}$ e $\mathrm{F}$, adotando-se o nível de 5\% de significância.

\section{Resultados e discussão}

As folhas apresentaram C, L e AF média de $25,15 \mathrm{~cm}$, $15,57 \mathrm{~cm}$ e $162,98 \mathrm{~cm}^{2}$ aos 21 DAE e $29,09 \mathrm{~cm}, 20,06 \mathrm{~cm}$ e $263,33 \mathrm{~cm}^{2}$ aos 50 DAE, respectivamente (TAB. 2).

Os resultados de regressão efetuados, relacionando a $\mathrm{AF}$ com $\mathrm{C}, \mathrm{L}, \mathrm{CL}$ e $(\mathrm{CL})^{2}$, indicam que todas as equações apresentadas permitiram obter estimativas satisfatórias da área foliar da batateira, com $\mathrm{R}^{2}$ acima de 0,75 , com exceção da equação exponencial, relacionando AF somente com $(\mathrm{CL})^{2}$ aos 21 DAE e das equações linear, exponencial e logarítmica relacionando $\mathrm{AF}$ com $\mathrm{C}$, aos $50 \mathrm{DAE}$, cujos coeficientes de determinação ficaram em torno de 0,68 (TAB. 3 ). 
Tabela 2 - Valores máximos, mínimos e médios do comprimento, largura e área foliar da cultivar Atlantic, aos 21 e 50 dias após a emergência (DAE). Viçosa, UFV, 2006

\begin{tabular}{lcccccc}
\hline \multirow{2}{*}{ Característica } & \multicolumn{3}{c}{ Valores aos 21 DAE } & \multicolumn{3}{c}{ Valores aos 50 DAE } \\
\cline { 2 - 6 } & Máximo & Mínimo & Médio & Máximo & Mínimo & Médio \\
\hline Comprimento $(\mathrm{cm})$ & 37,90 & 14,20 & 25,15 & 36,50 & 20,50 & 29,09 \\
Largura $(\mathrm{cm})$ & 25,10 & 8,60 & 15,57 & 23,80 & 12,00 & 20,05 \\
Área da folha $\left(\mathrm{cm}^{2}\right)$ & 379,00 & 47,00 & 162,98 & 517,00 & 106,90 & 263,33 \\
\hline
\end{tabular}

Tabela 3 - Característica determinada, modelo estatístico, coeficiente de determinação $\left(\mathrm{R}^{2}\right)$ e equação ajustada para a estimativa da $\mathrm{AF}$ em função das medidas de C, L, CL e (CL) ${ }^{2}$ da folha da cultivar Atlantic, aos 21 e 50 DAE. Viçosa, UFV, 2006

\begin{tabular}{|c|c|c|c|}
\hline \multicolumn{4}{|c|}{$21 \mathrm{DAE}$} \\
\hline Característica & Modelo estatístico & $\mathrm{R}^{2}$ & Equação estimada \\
\hline $\mathrm{C}$ & \multirow{4}{*}{ Exponencial } & 0,88 & $\mathrm{AF}=1,32686 \mathrm{e}^{0,0332561^{* *} \mathrm{C}}$ \\
\hline $\mathrm{L}$ & & 0,81 & $\mathrm{AF}=1,41231 \mathrm{e}^{0,0482282 * *} \mathrm{~L}$ \\
\hline CL & & 0,85 & $\mathrm{AF}=1,74464 \mathrm{e}^{0,0010152^{* * *} \mathrm{CL}}$ \\
\hline$(\mathrm{CL})^{2}$ & & 0,68 & $\mathrm{AF}=1,97324 \mathrm{e}^{0,000000915132 * *(\mathrm{CL}) 2}$ \\
\hline $\mathrm{C}$ & \multirow{4}{*}{ Logarítmico } & 0,82 & $\mathrm{AF}=266,605 \mathrm{Ln}^{* *}(\mathrm{C})-688,596$ \\
\hline $\mathrm{L}$ & & 0,76 & $\mathrm{AF}=240,528 \mathrm{Ln}^{* *}(\mathrm{~L})-488,943$ \\
\hline $\mathrm{CL}$ & & 0,86 & $\mathrm{AF}=137,982 \mathrm{Ln} * *(\mathrm{CL})-651,739$ \\
\hline$(\mathrm{CL})^{2}$ & & 0,86 & $\mathrm{AF}=68,9911 \mathrm{Ln} * *(\mathrm{CL})^{2}-651,739$ \\
\hline \multicolumn{4}{|c|}{$50 \mathrm{DAE}$} \\
\hline Característica & Modelo estatístico & $\mathrm{R}^{2}$ & Equação estimada \\
\hline $\mathrm{C}$ & \multirow{4}{*}{ Exponencial } & 0,69 & $\mathrm{AF}=1,53236 \mathrm{e}^{0,0297299 * * \mathrm{C}}$ \\
\hline $\mathrm{L}$ & & 0,83 & $\mathrm{AF}=1,58526 \mathrm{e}^{0,0404812^{* * \mathrm{~L}}}$ \\
\hline $\mathrm{CL}$ & & 0,84 & $\mathrm{AF}=1,92575 \mathrm{e}^{0,000794278^{* *} \mathrm{CL}}$ \\
\hline$(\mathrm{CL})^{2}$ & & 0,76 & $\mathrm{AF}=2,17299 \mathrm{e}^{0,000000589833^{* *}(\mathrm{CL}) 2}$ \\
\hline $\mathrm{C}$ & \multirow{4}{*}{ Logarítmico } & 0,68 & $\mathrm{AF}=499,699 \mathrm{Ln} * *(\mathrm{C})-1416,1$ \\
\hline $\mathrm{L}$ & & 0,80 & $\mathrm{AF}=459,071 \mathrm{Ln}^{* *}(\mathrm{~L})-1107,18$ \\
\hline $\mathrm{CL}$ & & 0,84 & $\mathrm{AF}=271,135 \mathrm{Ln} * *(\mathrm{CL})-1457,37$ \\
\hline$(\mathrm{CL})^{2}$ & & 0,84 & $\mathrm{AF}=135,567 \mathrm{Ln}^{* *}(\mathrm{CL})^{2}-1457,37$ \\
\hline
\end{tabular}

$\mathrm{AF}=$ área foliar; $\mathrm{C}=$ comprimento da folha; $\mathrm{L}=$ largura da folha; $\mathrm{CL}=$ produto do comprimento $\mathrm{x}$ largura; $(\mathrm{CL})^{2}=$ quadrado do produto do comprimento $\mathrm{x}$ largura; $\mathrm{DAE}=$ dias após a emergência das plantas. ** Significativo ao nível de $5 \%$ de significância pelo teste " $\mathrm{t}$ "

Os resultados de regressão linear relacionando a $\mathrm{AF}$ e as medidas de C, L, CL e (CL) $)^{2}$, aos 21 e 50 DAE, estão apresentados nas Figuras 1 e 2, respectivamente. Notase que, na avaliação efetuada aos 21 DAE, a área foliar da batateira foi mais precisamente estimada $\left(\mathrm{R}^{2}=0,92\right)$, usando o modelo linear com as medidas de comprimento e largura $\left(\mathrm{AF}=0,369335^{* *} \mathrm{CL}+10,6923\right)$. Da mesma forma, as medidas de comprimento e largura também foram mais precisas $\left(\mathrm{R}^{2}=0,86\right)$, na avaliação realizada aos $50 \mathrm{DAE}\left(\mathrm{AF}=0,470971^{* *} \mathrm{CL}-16,3432\right)$. 

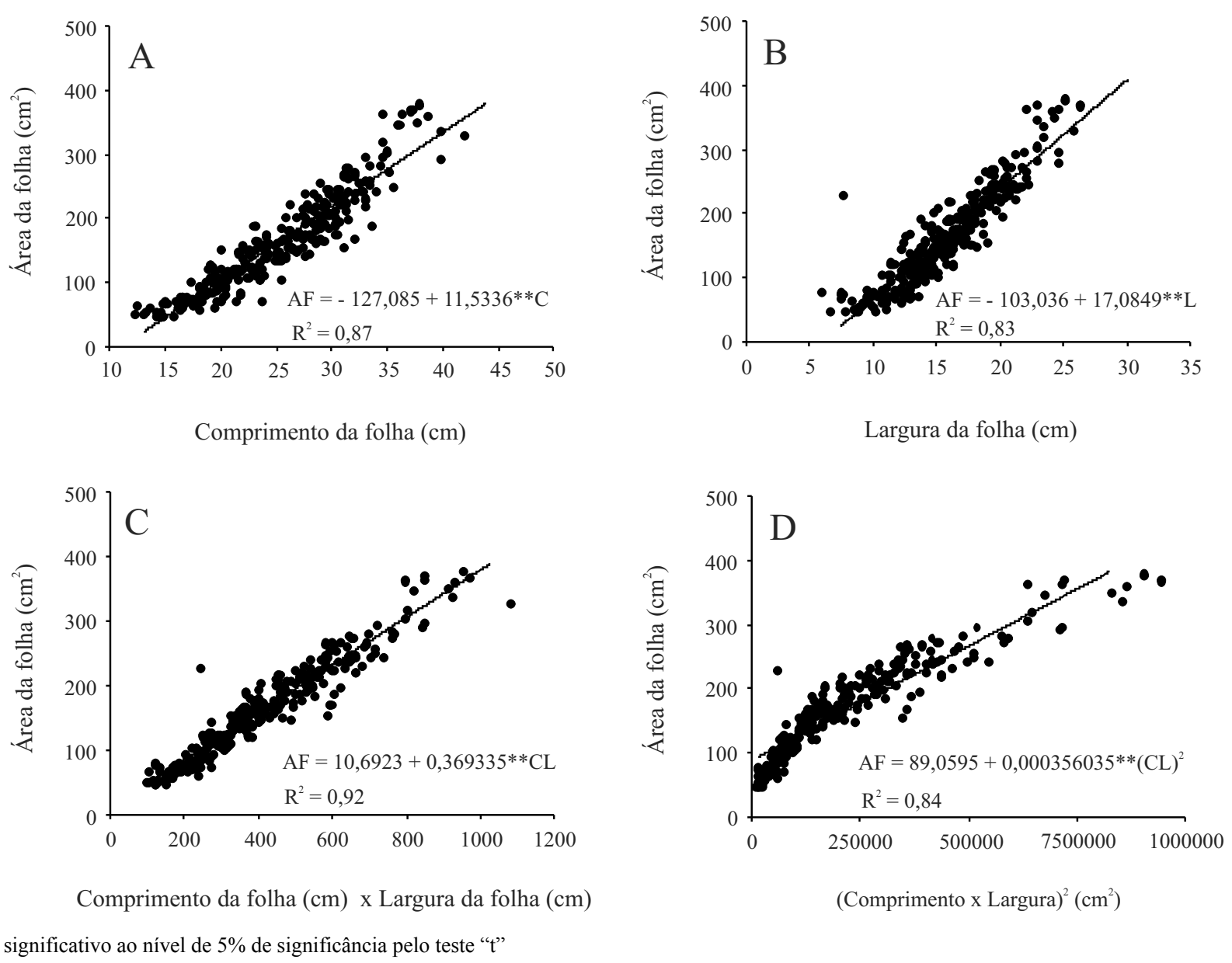

Figura 1 - Relação entre o comprimento (A), a largura (B), o produto do comprimento $\mathrm{x}$ largura (C) e o quadrado do comprimento $\mathrm{x}$ largura (D) com a área foliar da batateira, cultivar Atlantic, aos 21 DAE. Viçosa, UFV, 2006

Similarmente ao ocorrido com o modelo linear, a equação com o produto da largura pelo comprimento da folha proporcionou o maior valor de $\mathrm{R}^{2}$ para os modelos exponencial e logarítmico. Os maiores valores do coeficiente de determinação foram observados nas equações que utilizaram o produto do comprimento pela largura (CL), permitindo obter estimativas mais acuradas da área foliar da batateira. Estas equações apresentaram um coeficiente de determinação acima de 0,84 , indicando que, das variações existentes na área foliar, $84 \%$ podem ser explicadas por esses modelos (FIG. 1 e 2; TAB. 2). Em trabalho de Silva et al. (2008), independente do modelo estatístico, linear, exponencial ou logarítmico, a área da folha (AF) da batateira aos 21 e 56 dias, após a emergência, foi mais precisamente estimada $\left(\mathrm{R}^{2}=0,88\right)$, usando a combinação das medidas de largura (L) e comprimento (C) da folha ( $\mathrm{AF}=0,2798 * * \mathrm{LC}+71,267)$.

Pires et al. (1999), testando diferentes métodos para calcular a área foliar do morangueiro, por meio das dimensões das folhas, também obtiveram melhores resultados quando analisaram comprimento e largura conjuntamente. Strik e Proctor (1985) também concluíram que a utilização conjunta do comprimento e da largura melhor estimou a área dos folíolos de morangueiro. Corroborando com os demais autores, o mesmo foi observado por $\mathrm{Lu}$ et al. (2004), quando trabalharam com cultivares de taro, e concluíram que $\mathrm{o}$ modelo que inclui comprimento $\mathrm{x}$ largura da folha proporcionou maior consistência na estimativa da área foliar ao longo do desenvolvimento da folha do que modelos com apenas uma medida.

Segundo Campostrini e Yamanishi (2001), os modelos matemáticos estimadores de área foliar para figueira podem ser obtidos por diferentes medições do comprimento das folhas das plantas, ao longo da nervura principal. Já Schwarz e Kläring (2001) não recomendam a utilização da largura da folha, pois é mais difícil e consome mais tempo do que na 

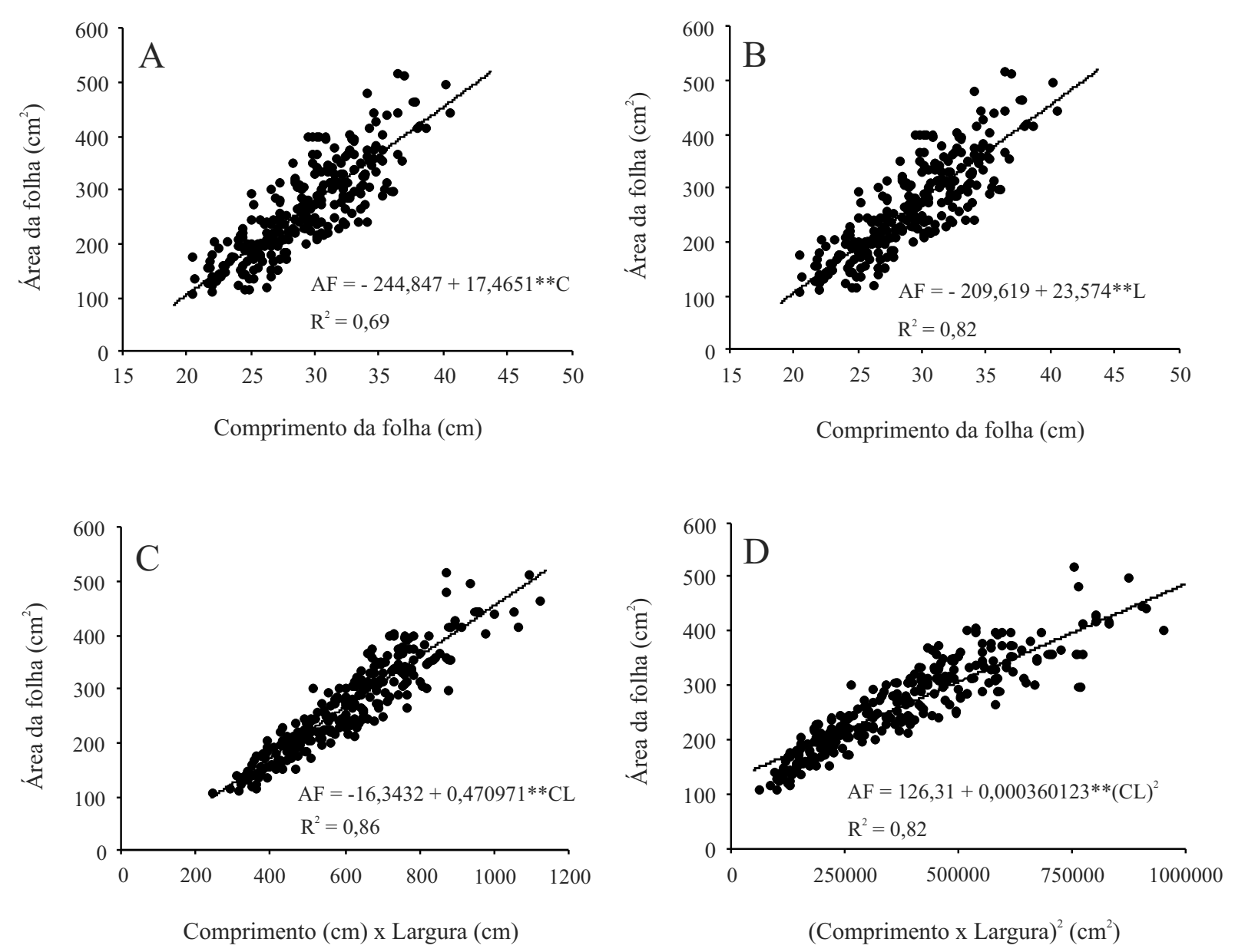

** - significativo ao nível de 5\% de significância pelo teste " $\mathrm{t}$ "

Figura 2 - Relação entre o comprimento (A), a largura (B), o produto do comprimento x largura (C) e o quadrado do comprimento $\mathrm{x}$ largura (D) com a área foliar da batateira, cultivar Atlantic, aos 50 DAE. Viçosa, UFV, 2006

determinação do comprimento, devido à necessidade do alinhamento dos folíolos, apesar de considerarem mais precisa a estimativa da área da folha utilizando a medida de largura. Porém, nos estudos com a cultura do meloeiro (NASCIMENTO et al., 2002) e do feijoeiro (QUEIROGA et al., 2003), a largura máxima foi considerada.

A maioria dos trabalhos disponíveis na literatura, de acordo com Monteiro et al. (2005), tem como objetivo principal a verificação de qual dimensão da folha ou do folíolo apresenta melhor correlação com a área da folha e da planta toda, sem dedicar muita atenção às diferenças de forma das folhas. Isso reflete na limitação prática inerente ao método que é a dificuldade de se realizar medidas em um número grande de folhas ou plantas. Por outro lado, a vantagem desse método é sua possibilidade de uso com um mínimo de recursos, além de ser utilizado como método não destrutivo.
A maior parte das equações obtidas pode ser empregada para estimar a área foliar da batata. Essas equações permitem com a medição no campo de comprimento ou largura ou ambas estimar a área foliar da planta.

\section{Conclusão}

Com os modelos propostos, as medidas de comprimento e largura das folhas são adequadas para estimar a área foliar da batata, cultivar Atlantic, em tempo real, de forma rápida e sem a necessidade de coletar a folha. Do ponto de vista prático, utilizando-se apenas uma medida para estimar a área foliar, com reduzida perda de precisão, recomenda-se utilizar a equação $\mathrm{AF}=11,5336^{* *} \mathrm{C}-127,085$ $\left(\mathrm{R}^{2}=0,87\right)$, aos 21 DAE ou a equação $\mathrm{AF}=23,574 * * \mathrm{~L}$ 209,619 $\left(\mathrm{R}^{2}=0,82\right)$, aos 50 DAE. 


\section{Agradecimentos}

À FAPEMIG e ao CNPq pelo apoio financeiro.

\section{Referências}

BIANCO, S.; PITELLI, R. A.; CARVALHO, L. B. Estimativa da área foliar de Cissampelos glaberrima usando dimensões lineares do limbo foliar. Planta Daninha, v. 20, n. 03, p. 353-356, 2002.

BIANCO, S.; PITELLI, R. A.; PITELLI, A. M. C. M. Estimativa da área foliar de Typha latifolia usando dimensões lineares do limbo foliar. Planta Daninha, v. 21, n. 02, p. 257-261, 2003.

CAMPOSTRINI, E.; YAMANISHI, O. K. Estimation of papaya leaf área using the cental vein length. Scientia Agricola, v. 58, n. 01, p. $39-42,2001$

DUARTE, D. J. et al. Estimativa da área foliar de Euphorbia heterophylla. Planta Daninha, v. 27, n. 03, p. 527-531, 2009.

EMPRESA BRASILEIRA DE AGRICULTURA E PECUÁRIA (EMBRAPA). Centro Nacional de Pesquisa de Solos (Rio de janeiro, RJ). Sistema brasileiro de classificação de solos. 3. ed. Brasília: Embrapa. Produção de informação; Rio de janeiro: Embrapa. Solos. 2006. 306 p.

FONTES, P. C. R. Diagnóstico do estado nutricional das plantas. Viçosa: UFV, 2001. 122p.

FONTES, P. C. R. Olericultura: teoria e prática. In: FONTES, P. C. R. Cultura da batata. Viçosa: UFV, 2005. p. 323-343.

FONTES, P. C. R.; ARAÚJO, C. Adubação nitrogenada de hortaliças: princípios e práticas com o tomateiro. Viçosa: UFV, 2007. 148 p.

KOEPPEN, W. Climatologia. Buenos Aires: Gráfica Panamericana, 1948. $478 \mathrm{p}$.

KVET, J.; MARSHALL, J. K. Assessment of leaf area and other assimilating plant surfaces. In: SESTÁK, Z.; EATSKY, J.; JARVIS, P. G. Plant photosynthetic production: manual of methods. Netherlands: Dr. W. Junk N.V. Publ., 1971. p. 517-555.

LARCHER, W. Ecofisiologia vegetal. São Paulo: Rima. 2000. 531p.
LU, H. Y. et al. Comparison of different models for nondestructive leaf area estimation in taro. Agronomy Journal, v. 96, n. 02, p. 448-453, 2004

MARSHALL, J. K. Methods of leaf area measurement of large and small leaf samples. Photosynthetica, v. 02, p. 41-47, 1968.

MONTEIRO, J. E. B. A. et al. Estimação da área foliar do algodoeiro por meio de dimensões e massa das folhas. Bragantia, v. 64 , n. 01, p. $15-24,2005$.

NASCIMENTO, I. B. et al. Estimativa da área foliar do meloeiro. Horticultura Brasileira, v. 20, n. 04, p. 555-558, 2002.

PARTELLI, F. L. et al. Estimativa da área foliar do cafeeiro conilon a partir do comprimento da folha. Revista Ceres, v. 53, n. 306, p. 204-210, 2006.

PIRES, R. C. M.; FOLEGATTI, M. V.; PASSOS, F. A. Estimativa da área foliar do morangueiro. Horticultura Brasileira, v. 17, n. 02, p. 86-90, 1999.

QUEIROGA, J. L. et al. Estimativa de área foliar do feijão-vagem (Phaseolus vulgaris L.) por meio da largura máxima do folíolo central. Horticultura Brasileira, v. 21, n. 01, p. 64-68, 2003.

RIBEIRO JÚNIOR, J. I. Análises estatísticas no SAEG. Viçosa: UFV, 2001. $301 \mathrm{p}$

SILVA, M. C. C.; FONTES P. C. R.; VIANA R. G. Estimativa da área da folha da batateira utilizando medidas lineares. Horticultura Brasileira, v. 26, n. 01, p. 83-87, 2008.

SILVA, N. F. et al. Modelos para estimar a área foliar de abóbora por meio de medidas lineares. Revista Ceres, v. 45, n. 259 , p. $287-291,1998$.

SCHUWARZ, D.; KLARING, H. Allometry to estimate leaf area of tomato. Journal of Plant Nutrition, v. 24, n. 08, p. 1291-1309, 2001.

STRIK, B. C.; PROCTOR, J. T. A. Estimating the area of trifoliate and uneqully imparipinnate leaves of strawberry. HortScience, v. 20, n. 06, p. 1072-1074, 1985.

TAIZ, L.; ZEIGER, E. Fisiologia Vegetal. 3. ed. Porto Alegre: Artmed, 2004. 719 p.

TERUEL, D. A. Modelagem do índice de área foliar de cana-de-açúcar em diferentes regimes hídricos. 1995. $93 \mathrm{f}$. Dissertação (Mestrado em Agronomia) - Escola Superior de Agricultura de Luiz de Queiroz, Piracicaba. 